\title{
Interactive comment on "A standardized database of Marine Isotopic Stage 5e sea-level proxies on tropical Pacific Islands" by Nadine Hallmann et al.
}

\author{
Blake Dyer (Referee) \\ blakedyer@uvic.ca
}

Received and published: 9 April 2021

This work by Hallman et al. represents a significant effort to organize and contextualize decades of MIS 5e Pacific Island data. This effort will be invaluable at facilitating future research on MIS 5e sea level and Pacific Island record of MIS 5e. I would confidently recommend this database and manuscript, as is, to any student or researcher looking for a starting point on MIS $5 \mathrm{e}$ in the Pacific. However, upon reflecting on the manuscript and previous comments in the discussion, it is clear that some of the detail is lost in the conversion of decades of field work to site-by-site RSL interpretations.

I have some reservations that the 84 summary RSL indicators will be used without any consideration of the assumptions made in the conversion. My understanding is that 
the authors have followed well documented approaches to estimate paleo-RSL, and that these approaches are standardized throughout the WALIS database. I suspect experts will have no trouble navigating where each paleo-RSL number comes from, but the text could offer more guidance and caution to non-experts. I note that the authors have clearly considered this issue and adressed it in the text, so I only have a few minor suggestions that they may wish to incorporate:

First, if we consider a single site as an example: 5.1 French Polynesia. The text presents a logical narrative from data (ages and species or species assemblages) to RSL. Paraphrasing from Lines 356-359: ..past workers reported an elevation of Leptoria at $3.85 \mathrm{~m}$, Leptoria lives today down to $7.5 \mathrm{~m}$, and so paleo-RSL is $8.75+/-2.3$. As a non-expert or someone not intimately familiar with WALIS, trying to unravel the conversion to paleo-RSL is impossible. Moreover, the details will differ some for each of the 84 summary sites. I suggest that the introduction to section 5 include a general workflow from data to paleo-RSL interpretations. This small addition could include a reference to the online WALIS documentation or any other manuscripts within this special issue that may document more fully the process. An additional sentence after line 44 in the introduction could be helpful too, although my next comment is related to this possible approach.

Second, the authors address the uncertainties and data quality extensively in section 6.6. I do believe this discussion is appropriately detailed. However, I strongly agree with the comment from Clark that the information seems to come too late in the manuscript. The authors reply to that comment mentions that the format is fixed, so I would encourage the authors to include in the introduction a very brief summary of the uncertainties and challenges associated with estimating paleo-RSL from the data in the database. This section could refer readers to section 6.6 for a more complete discussion.

Interactive comment on Earth Syst. Sci. Data Discuss., https://doi.org/10.5194/essd-2020-261, 2020. 